\title{
Soil solution calcium carbonate equilibrium as a driver of soil organic matter and heavy metals transfer and turnover in focus of humic substances soil fertility effect
}

\author{
Minkina T.M. ${ }^{1}$, Glinushkin A.P. ${ }^{2}$, Kalinitchenko V.P. ${ }^{2,3}$, Makarenkov D.A. ${ }^{4}$, \\ Zinchenko V.E. ${ }^{5}$, Chernenko V.V. ${ }^{6}$, Chelnokov V.V. ${ }^{7}$, Mandzhieva S.S. ${ }^{1}$, Sushkova S.N. ${ }^{1}$, \\ Il'ina L.P. ${ }^{8}$, Chaika V.A. ${ }^{4}$ \\ ${ }^{1}$ Southern Federal University, Rostov-on-Don, Russia, tminkina@mail.ru \\ ${ }^{2}$ Russian Research Institute of Phytopathology, Bolschije Wjasjomy, Russia \\ ${ }^{3}$ Institute of Fertility of Soils of South Russia, Persianovka, Russia \\ ${ }^{4}$ Institute of Chemical Reagents and High Purity Chemical Substances of National Research \\ Centre "Kurchatov Institute", Moscow, Russia \\ ${ }^{5}$ Federal Rostov Agrarian Research Center, Rassvet, Rostov Region, Russia \\ ${ }^{6}$ Russian Research Institute of Viticulture and Winemaking, Novocherkassk, Federal Rostov \\ Agrarian Research Center, Rassvet, Rostov Region, Russia \\ ${ }^{7}$ Mendeleev University of Chemical Technology of Russia, Moscow, Russia \\ ${ }^{8}$ Southern Scientific Center of Russian Academy of Sciences, Rostov-on-Don, Russia
}

Keywords: Soil organic matter, C, N, P, and heavy metals turnover, soil solution calcium carbonate equilibrium, humic substances management success

doi: 10.36291/HIT.2019.minkina.121

The knowledge problem of development is to identify critical boundary conditions of ambiguity, complexity, equivocality, and uncertainty as an ecological analytical construct, and apply new innovative possibilities for strategic improved landscape management in focus of biogeochemical cycling and resources growth in every part of biosphere, in particular in vast low productive terrains of dry steppe. It is pertinent that the uptake capacity of land-based soil carbon (C) sinks be enhanced through targeted management. However, the best management practices should not be focused on $C$ sequestration from the atmosphere, or irreversible subsoil and geological $\mathrm{C}$ burial. Simultaneous $\mathrm{C}$ global balance stimulation and $\mathrm{C}$ biological phase enrichment are strongly needed. An attempt to have a carbon fixation effect for the sake of net hydrogen production and negative balances of $\mathrm{CO}_{2}$ emissions will turn to the atmosphere water vapor excess after hydrogen burning. Water vapor is more dangerous greenhouse gas than $\mathrm{CO}_{2}$. Soil organic matter (SOM) is declining in most agricultural ecosystems, impacting multiple ecosystem services. The nature of $C$, nitrogen $(N)$, phosphorus $(P)$, and heavy metals (HMs) turnover is critical for the global biogeochemical cycling. Currently, the stability of existing artificial steppe ecosystems is extremely weak, and therefore the dryland $C$ balance is switching from soil $C$ uptake to soil $\mathrm{C}$ efflux. The uncontrolled water, mineral and organic matter transfer is an adverse consequence of the standard ecosystems management. Temporal dynamics of soil structure alteration is deemed to be important for essential ecosystem functions but very little is known about it. The state and transfer of SOM, P and other nutrients, and microelements including HMs are closely linked to the content and state of carbonates in soil and in soil solution. Carbonates, as a part of soil minerals, live and decaying organic matter cause the formation of associated ions and complexes of carbonates, SOM, P, microelements, and HMs. Organic matter forms films on the primary carbonates surface, reducing carbonate sedimentation. Thus, the range of mobility, transfer, and bioavailability of organic matter, $\mathrm{P}$, microelements, and HMs is vast and uncertain. The cause of soil solution excess saturation with calcium carbonate, as well as the transfer and accumulation of organic matter, and HMs is the ions association and formation of mineral and organic complexes in soil solution. The properties, structure, and soil solution calcium carbonate equilibrium (CCE) is under the influence of ions activity and determines the dynamics of material composition, migration, and accumulation of mineral and organic matter into the soil continuum aggregate system, determining the humic substances management success under the influence of CCE. 